\title{
PLACEMAKING DE LOUIS I. KAHN
}

\author{
Marta Pieczara \\ Politechnika Poznańska, Wydział Architektury, Instytut Architektury \\ i Planowania Przestrzennego, Politechnika Poznańska \\ Faculty of Architecture, Institute of Architecture and Planning, Poznań University of Technology \\ e-mail: marta.pieczara@alumni.epfl.ch
}

\begin{abstract}
Résumé. Défini comme «la fabrication des lieux», le placemaking repose non pas seulement sur un vocabulaire architectural recherché, mais aussi sur une union harmonieuse de l'architecture et du site. Pour Louis I. Kahn, le rôle que le territoire joue dans ses projets contribue aussi au développement de sa méthode. Les différents endroits dans lesquels il construit lui offrent une source des réflexions générales. Tout au long de sa carrière, Kahn élabore une série de principes que nous pouvons définir comme ses «outils» du placemaking et qui sont inhérents à sa méthode.
\end{abstract}

Mots-clés: placemaking, lieu, territoire, caractère urbain, méthode de projet, mise en place.

\section{INTRODUCTION}

Le terme de placemaking, qui signifie littérairement la fabrication des lieux, désigne l'art de créer les espaces uniques qui attirent des êtres humains en leur offrant le sentiment d'appartenir à la société. «Les lieux sont des espaces dont nous nous rappelons, dont nous nous soucions et qui font partie de notre vie». [Lyndon, Moore, 1994, p.xii] Au travers de placemaking, les architectes ont ainsi pouvoir de «situer une personne (utilisateur) dans le contexte collectif et individuel». [Goldhagen, 2001, p.207]

La préoccupation de concevoir les lieux uniques et non pas seulement l'architecture domine l'œuvre mature de Louis I. Kahn. Cependant, ce qui nous intéresse le plus, c'est le fait que placemaking suppose une réunion harmonieuse de l'architecture et du lieu. [Goldhagen, 2001, p.205] Nous pouvons ainsi présumer que, au-delà d'une réalité sociale, l'architecte prend en considération aussi de différents aspects de l'environnement: la géographie, la végétation et l'histoire du lieu, pour en donner quelques exemples fondamentaux.

Aux yeux de Louis I. Kahn, le processus de projet, ou alors le test de la validité de la forme, consiste à faire face au mesurable, aux contingences. Parmi ces conditions mesurables, les éléments de l'environnement sont beaucoup plus importants pour l'architecte que le budget et le programme. Telle est l'indication que nous donne August Komendant, l'ingénieur qui a travaillé avec Kahn pendant dix-huit ans. D'après ses dires, «the timing and assignment to the students very often ran parallel to his own assignments. [...] Kahn's assignment to students was not just to design a building but a building in an environmental setting». [Komendant, 1975, p.177] Si l'architecte donnait aux étudiants certains des sujets sur lesquels il travaillait personnellement, en soulignant l'importance de l'environnement, nous pouvons présumer que ce dernier jouait un rôle aussi important dans ses propres projets. Et, par conséquent, les idées de l'architecte étaient vérifiées dès lors qu'elles avaient pris leur orientation dans le contexte et lorsqu'elles étaient matérialisées au travers du processus de design. Il s'ensuit une question concernant le rôle que les éléments contextuels peuvent jouer dans ce processus, envisagé par Kahn comme test de la validité de la forme. Le but de cet écrit sera donc d'examiner sa méthode de travail avec un regard particulier sur l'intégration de ses projets dans leur cadre environnemental. La question à laquelle on essaie de répondre est sur quels éléments du lieu Kahn s'interroge-t-il pour mettre en œuvre le placemaking? Et ensuite, quel est le rôle de ces éléments ? Est-ce qu'ils peuvent inspirer l'architecte, l'influencer? L'objectif de ce texte est ainsi d'identifier et 
de définir ceux parmi les outils de la conception spatiale utilisés par Louis I. Kahn qui sont liés à l'analyse du territoire. Nous allons les appeler ses outils de placemaking. Pour mieux comprendre leur fonctionnement, nous allons suivre leur évolution au travers de différents projets.

\section{OBJETS D’ÉTUDE}

Le corpus d'étude se limite aux six projets réalisés, sélectionnés en fonction de leur caractère public et institutionnel en relation étroite avec l'espace public (la forme urbaine), jusqu'à offrir un certain symbolisme. Les projets choisis sont: les laboratoires RichardsGoddard de Philadelphie, PA (1957-64); le Salk Institute de La Jolla, CA (1959-65); First Unitarian Church de Rochester, NY (1959-67); Erdman Hall Dormitories de Bryn Mawr, PA (1960-65); Phillips Exeter Academy Library d'Exeter, NH (1965-71) et Kimbell Art Museum de Forth Worth, TX (1966-72).

Toutes ces six œuvres sont élaborées après le projet de Trenton Bath House (19541957) au cours duquel Kahn conçoit une manière universelle de répondre à la question de la hiérarchie spatiale. Il s'agit du concept opératoire des espaces servants et servis (ang. serving and served spaces) qui est devenu sa première méthode de conception spatiale. S'ensuivent d'autres concepts théoriques que Kahn développe au cours de sa carrière. En tant que professeur, Kahn les explique aux étudiants à Yale et, plus tard, à Philadelphie. En outre, tous les projets sélectionnés ont le caractère public et institutionnel. Ce choix se justifie par l'intérêt que ce texte porte à la notion du placemaking et à la responsabilité sociale dont Kahn se charge. Puisqu'il s'adresse au grand public, le placemaking s'applique plutôt aux institutions qu'à d'autres fins. De ce fait, le contenu contextuel des projets de Kahn devrait être plus lisible dans le cas des institutions. En outre, les dates d'élaboration des projets choisis sont également importantes. C'est à partir des années 50 que Kahn commence de concevoir des lieux uniques, ce qui entraîne la référence aux principes du placemaking.

Principalement, le corpus d'étude se constitue des dessins, de la littérature, des archives, des cartes, de l'imagerie satellite et de la photographie aérienne.

\section{MÉTHODE}

Pour chacun des six projets sélectionnés, la recherche procédait tout d'abord par l'analyse de l'environnement et de son histoire. Dans cette catégorie, le premier sujet étudié était le territoire: sa morphologie, l'hydrographie, la végétation, le réseau de transport ainsi que son climat. Ensuite, nous nous sommes concentrés sur le caractère urbain du lieu. En relation avec l'histoire de la ville, nous avons suivi le développement de sa structure urbaine. Un regard plus attentif est porté sur les espaces représentatifs et sur les espaces verts. Nous nous sommes intéressés aussi à l'architecture du lieu, aux styles et matériaux prédominants ainsi qu'à la composition du quartier.

Deuxièmement, nous avons abordé toutes les questions concernant le développement de l'œuvre, comprenant toutes les phases du projet: de l'étape conceptuelle à la réalisation. Les plus importantes parmi les notions abordées sont: le concept; le type et l'archétype; la mise en place et le choix de l'entrée; le seuil; la distribution; l'usage; l'application du concept des espaces servants et servis; la lumière; la structure; l'ordre constructif et le choix des matériaux.

Quant à l'instrument utilisé, outre l'analyse écrite, des études graphiques ont été effectuées. Ce travail de dessin a pour but de localiser le projet concerné dans son territoire, ce qui était peu analysé dans les publications existantes sur l'architecte. Dans la mesure du possible, nous avons essayé de recréer l'état du contexte lors de l'édification. 


\section{RÉSULTATS}

Dès que la partie analytique a était faite, nous avons pu procéder à l'analyse des rapports entre l'œuvre et son contexte. Sur la base de notre recherche, nous avons identifié les différents outils que l'architecte a mis en œuvre pour réaliser les lieux uniques:

\section{APPROPRIATION DE L'ÉCHELLE}

Pour Louis I. Kahn, l'emplacement d'un projet dans le territoire n'est pas une décision immédiate ni définitive. Au contraire, l'adéquation du projet au site est, pour lui, un long processus d'apprentissage. Au cours de ce processus, l'architecte met en œuvre une méthode d'appropriation de l'échelle du lieu. L'objectif de cette longue étude est d'identifier et de s'approprier dans la mesure du possible les richesses du territoire. En d'autres mots, Kahn veut faire sien le territoire. Il choisit les lieux les plus importants des sites pour les transformer ensuite en termes de concepts architecturaux. L'appropriation des lieux particuliers, spécifiques, est pour Kahn l'un des moyens de créer des œuvres uniques.

La volonté de tester le potentiel du lieu détermine le développement du projet, surtout dans sa phase initiale. Souvent, cette volonté amène l'architecte à expérimenter un hors d'échelle, comme dans le cas du Salk Institute (Ill. 1). Pourtant, cette «déformation» de l'échelle lui sert de méthode pour acquérir une compréhension du lieu. L'intérêt qu'il porte à la qualité du site coïncide avec la recherche de l'adéquation entre cette qualité et le sens de l'objet architectural. Pour le Salk Institute de La Jolla, la reconnaissance archétypique du site apporte le concept de trois groupes fonctionnels: les laboratoires sur la plaine, le Meeting House sur la falaise et les résidences au bord du canyon (Ill. 1).

La décroissance de l'échelle du projet au fil des phases est simultanée avec l'étude du territoire. Kahn commence sa première approche de l'appropriation du lieu avec une vision globale, dans laquelle il cherche à identifier des éléments qui peuvent être transformés en termes de concepts architecturaux. De l'autre côté, ces mêmes éléments peuvent lui servir de repères d'échelle, tout en étant le fondement de la compréhension du lieu. C'est par exemple le cas du musée Kimbell où à l'intention de croiser la rue Will Rogers East correspond un étirement de la composition d'une limite de la parcelle à l'autre. Ensuite, une réduction graduelle d'échelle du bâtiment, opérée toujours par rapport à l'ancienne rue, mène à la version finale (Ill. 2).

\section{ANALYSE DU CARACTÈRE URBAIN}

La documentation du territoire recueillie par l'architecte constitue en même temps une riche source d'informations sur le caractère urbain du lieu. La forme urbaine constitue pour Louis I. Kahn une source d'inspiration et, avant tout, des repères importants qui déterminent souvent les premiers principes du projet. Avant tout, Kahn s'intéresse à la typologie de l'architecture locale. Simultanément, il cherche des règles de composition dans la forme urbaine du lieu et s'interroge sur la question de l'alignement et sur le concept d'îlot. Conjointement, il réfléchit au gabarit.

Pour la bibliothèque d'Exeter, par exemple, Kahn reprend le type caractéristique local pour composer le nouveau bâtiment selon le même ordre: le socle, les trois niveaux et le couronnement. Malgré la différence considérable du gabarit, la bibliothèque renvoie ainsi au caractère historique du lieu, y compris l'échelle. Pour le musée Kimbell de Fort Worth, ensuite, l'architecte interprète le type pavillonnaire caractéristique du quartier pour proposer un bâtiment de même typologie. Simultanément, la forme urbaine inspire les deux caractères différents d'entrée que l'architecte propose: «une entrée d'arbres» du côté du parc et une «back door» du côté de la rue. 


\section{CHOIX D’ACCÈS}

Pour parvenir au choix de l'entrée, Kahn s'inspire du contexte de plusieurs manières. Tout d'abord, il étudie les différentes possibilités d'accès. Il identifie les routes principales et des rues secondaires qui desservent le site et il choisit l'endroit d'accès pour les voitures. S'ensuivent les décisions concernant la localisation du parking et de l'accès des services. Conjointement, le choix de l'entrée principale piétonne dépend de plusieurs autres aspects. Outre le programme qui contient souvent les indications concernant le nombre souhaité des entrées ainsi que leur caractère, l'architecte examine attentivement tous les éléments du contexte qui peuvent indiquer l'entrée et il n'hésite pas à en profiter pour perfectionner le projet. Par exemple, le fonctionnement très apprécié du musée Kimbell est étroitement lié à la façon dont l'architecte a utilisé la pente pour séparer les deux accès différents (Ill. 3).

\section{USAGE DE LA PENTE}

Aux yeux de l'architecte, l'usage de la pente constitue l'un des moyens de s'approprier les lieux privilégiés du site, de profiter le plus possible de ses qualités. L'interdépendance du bâtiment avec la morphologie du territoire est donc, pour Kahn, d'une importance fondamentale pour réussir le placemaking. Louis I. Kahn n'hésite pas de se servir de la topographie du site pour créer une architecture qui s'intègre au territoire, qui en bénéficie au maximum et qui l'exprime en même temps. C'est pour exprimer la pente qu'il choisit de mettre la résidence Erdman de Bryn Mawr exactement sur la cassure du terrain. Simultanément, l'architecte se sert de la dénivellation naturelle pour modifier la perception de l'échelle du bâtiment. Identique à la résidence Erdman, la coupe du musée Kimbell de Fort Worth est également conçue pour exprimer la pente (Ill. 3). Dans ce projet, l'architecte s'inspire de la morphologie du terrain pour définir les principes de la distribution et de la hiérarchie spatiale (un niveau représentatif et un niveau servant).

\section{CHOIX DU TYPE: CENTRALITÉ OU ENFILADE?}

Avant de faire le choix du type, l'architecte prend en considération non seulement les modèles architecturaux sous-jacents au thème du projet, mais aussi certains critères contextuels. Outre la vision conceptuelle relative au dessein de l'objet, le choix du type provient ainsi d'une coïncidence de plusieurs éléments, comme la localisation et la forme du site, ses rapports avec l'environnement urbain ou encore les inspirations locales. Cependant, ce qui est le plus important, Louis Kahn s'intéresse énormément à la notion d'idéalité du type par rapport au lieu. Par une mise en contexte réfléchie, l'architecte cherche à avaliser le type choisi.

Pour démontrer cette proposition, il faut d'abord classifier les projets sélectionnés selon leurs types architecturaux. Les trois groupes typologiques essentiels qu'on peut distinguer sont fondés soit sur la notion de centralité, soit sur la notion d'enfilade, ou encore sur une combinaison de ces deux notions. Parmi les projets analysés, le type parfaitement central est représenté par la bibliothèque d'Exeter, tandis que le musée Kimbell constitue un exemple de l'enfilade.

Pour la bibliothèque d'Exeter, d'abord, la localisation dans les limites de l'espace vert central du campus conforte le choix du type central. De ce type provient ensuite le caractère distributif périphérique du bâtiment. La notion de centralité, qui apparaît tout au début du projet de la bibliothèque, exprime non seulement les principes conceptuels du projet, mais aussi le caractère de sa localisation. Dans le cas du musée Kimbell, le choix du type fondé sur l'enfilade facilite l'exploitation du site en pente. Notamment, il permet à l'architecte de profiter pleinement de la morphologie du territoire pour définir le caractère distributif transversal du musée et pour renforcer la liaison entre son projet et le contexte urbain. Malgré toute la différence typologique, la bibliothèque d'Exeter et le musée Kimbell illustrent ainsi une même 
démarche que l'architecte fait pour assurer des bons rapports entre ses projets et leurs environnements. Dans ces deux exemples, les deux lieux singuliers amènent l'architecte à choisir deux types différents. Le choix du type est, pour Louis Kahn, le premier pas pour exprimer sa compréhension du territoire à travers un projet.

\section{PAYSAGE}

Aux yeux de Louis I. Kahn, le paysage, compris comme expression de la morphologie du territoire revêtu de végétation, est l'un des éléments les plus importants du lieu. Nous pouvons observer que le terme du paysage rassemble les différentes formes de la représentation du territoire. Selon le caractère de la zone, le paysage peut être urbain, naturel ou mixte. La manière de voir la nature est aussi importante que la qualité du paysage. Outre la morphologie du terrain et le caractère urbain, Louis Kahn concentre son attention sur la qualité de la végétation. Il s'intéresse énormément aux différents essences d'arbres, à leur feuillage et à leur couleur.

Pour Louis Kahn, le paysage constitue l'un des éléments fondamentaux de ses projets. D'entre les six bâtiments analysés, tous peuvent servir d'exemple. Pour les laboratoires médicaux de Philadelphie, d'abord, le jardin botanique existant contribue à la notion de l'îlot qui est l'un de premiers principes du projet. À Exeter, la bibliothèque s'inscrit entre les grands arbres du parc dans lequel le bâtiment se situe. Avec l'architecture du lieu, la végétation forme l'environnement de référence pour le projet de Kahn. Pour l'église unitarienne de Rochester, ensuite, l'architecte se concentre sur la question de la vue de l'église depuis des différents endroits du site et des environs. Des différentes plantations autour de l'église sont prévues pour modifier la visibilité du bâtiment projeté. Pour le musée Kimbell, les deux rangées d'arbres de l'ancienne rue supprimée constituent l'élément principal de l'aménagement du site. Conjointement, la notion du jardin influence le choix de l'entrée représentative et la définition du caractère distributif. Pour la résidence de Bryn Mawr, ensuite, Louis Kahn se sert du parc préexistant pour donner l'air naturel à l'emplacement du projet. Pour le Salk Institute de La Jolla, enfin, la notion de regarder le paysage influence la forme et la disposition des ouvertures. À la même notion correspond également l'idée de la plaza centrale qui joue un rôle crucial en termes d'appropriation du paysage. Aux yeux de l'architecte, la plaza constitue une scène pour contempler la vue sur le canyon et sur l'océan. À travers cet espace Kahn pense théâtraliser le paysage (Ill. 4).

\section{SOLEIL ET LUMIÈRE}

Pour Louis I. Kahn, l'intensité du soleil constitue un élément du territoire pas moins important que sa morphologie. L'étude de son importance nécessite pourtant un classement par région géographique. Pour généraliser, nous pouvons distinguer trois zones principales dans lesquelles l'architecte a travaillé: le nord-est des États-Unis, le sud-ouest des États-Unis et les zones tropicales ou subtropicales du monde.

L'observation de la lumière du lieu est, pour Louis I. Kahn, le fondement pour développer les différents outils de contrôle. Tout d'abord, la lumière des villes de la Nouvelle Angleterre, qui est comparable à celle des villes italiennes, pousse l'architecte à étudier les possibilités de modifier l'ensoleillement des espaces à travers l'ouverture. Kahn différencie la lumière au moyen de différents types d'ouverture, de leur taille et, enfin, à travers des installations, comme des jalousies. Simultanément, il s'intéresse considérablement à la question de la fonctionnalité des espaces de l'ouverture. Dans ses premiers projets réalisés au nord-est des ÉtatsUnis, l'architecte cherche ainsi un rapport entre l'ouverture et l'activité de l'espace. Pour les laboratoires médicaux Richards de Philadelphie, par exemple, Kahn envisage une relation entre les bureaux des chercheurs et les grandes ouvertures rectangulaires. Dans les projets suivants, il relie la question d'éclairage avec le concept opératoire des espaces servants et 
servis et développe sa vision de l'espace servant qui cherche à maîtriser la lumière. Pour l'église unitarienne de Rochester, l'architecte entreprend un discours concernant la fonctionnalité de l'espace de l'ouverture qui aura des conséquences importantes pour ses travaux futures. Notamment, il envisage d'exploiter la lumière au moyen d'un mur extérieur en forme d'une série de niches dans lesquelles il installe des postes de lecture. Sur la base de cette idée, Kahn développe plus tard le concept des postes de travail alignés sur la façade du bâtiment, comme par exemple dans la bibliothèque d'Exeter et dans la résidence de Bryn Mawr.

Tandis que la périphérie du bâtiment se caractérise par l'entrée directe du soleil, les espaces centraux se voient attribuer une lumière zénithale indirecte se projetant sur les surfaces intérieures des murs. Ce caractère de la lumière correspond au caractère représentatif et à l'usage collectif de ces espaces. Kahn pense exprimer par ce choix leur importance symbolique. En d'autres mots, la distinction de la lumière constitue pour Louis Kahn un moyen d'exprimer les différents caractères des espaces. La lumière zénithale est utilisée, par exemple, dans le sanctuaire de l'église unitarienne de Rochester, dans les espaces centraux de la résidence de Bryn Mawr et dans le vide central de la bibliothèque d'Exeter.

\section{FAÇADE. CHOIX DES MATÉRIAUX}

Pour résumer la question du choix des matériaux, il faut d'abord identifier les deux facteurs importants qui s'y superposent. L'un concerne la réponse à l'environnement, tandis que l'autre est lié au choix de l'ordre structurel. Souvent, Louis Kahn se sert de matériaux différents pour montrer le rôle primaire ou secondaire des éléments structuraux. Tandis qu'un matériau représente la structure portante, l'autre s'applique à la structure portée. Il est important pour lui de rendre visible cette distinction à travers le choix des ouvertures. En règle générale, Kahn utilise les ouvertures pour séparer la structure porteuse de la structure portée. Aux deux matériaux fondamentaux que l'architecte choisit pour la structure primaire et secondaire s'ajoute souvent un matériau de parement.

Constituant la façade du bâtiment, le matériau de parement, ou parfois de la structure secondaire, est sélectionné pour rapprocher le nouveau bâtiment à son environnement. Avant tout, Kahn s'interroge sur le type de matériau commun au lieu et sur sa tonalité. Ainsi, Kahn choisit la brique pour les lieux où ce matériau est couramment utilisé, comme par exemple dans le nord-est des États-Unis ou sur le sous-continent indien. Deuxième exemple: dans le quartier pavillonnaire de Fort Woth où plusieurs institutions sont réalisées en calcaire, Kahn décide d'exprimer le caractère institutionnel du musée Kimbell par le travertin. Ou encore, dans le cas du Salk Institute, Kahn fait tout pour ajuster la tonalité du béton à l'ambiance de la côte du Pacifique. Le choix définitif du parement n'est pas tout simplement une conséquence directe des préférences personnelles ni d'une évaluation subjective de la valeur d'un matériau. À chaque fois, la sélection des matériaux procède d'une analyse du contexte, où le caractère du lieu est un critère important. Le traitement de la façade, qui sert à maintenir des relations avec le lieu, évoque une notion sous-jacente de l'opposition extérieur - intérieur.

\section{CONCLUSION}

Pour Louis I. Kahn, la maîtrise du placemaking coïncide avec une volonté de s'approprier les sites: leur géographie, leur topographie, leur paysage. Pour créer des lieux uniques, l'architecte recourt à ce qu'il a appris pendant un voyage en Italie, où les villages côtiers lui ont servi de premier exemple de lieux. Prenant en considération la qualité de la lumière naturelle, il cherche à harmoniser l'architecture avec le site. Pour atteindre l'effet désiré, il étudie la relation entre territoire et bâtiment non seulement par le dessin, mais aussi au moyen de maquettes. Pour le Salk Institute, par exemple, Kahn commande des maquettes à différentes échelles. L'une des maquettes, représentant le site avec la falaise de la côte Pacifique, permet à 
l'architecte d'étudier la qualité paysagère du projet. Loin du site, dans l'atelier de Philadelphie, cette maquette rend compréhensibles certaines références historiques que le projet évoque. Ainsi, par exemple, la référence à l'Acropole n'est pas seulement une inspiration formelle. Elle englobe également l'unité des bâtiments avec leur localisation; un élément que l'architecte érigera en concept.

Les différents outils de placemaking que Louis Kahn engage pour créer les lieux uniques ont évolué sous l'effet de son expérience. D'un projet à l'autre, l'architecte perfectionne sa méthode et il définit une série de règles selon lesquelles il agit. À l'instar des principes qui s'appliquent à la conception spatiale, ceux qui concernent l'appropriation du territoire jouent un rôle significatif dans le développement de l'œuvre de Louis I. Kahn. L'importance que l'architecte leur attribue, même si moins évidente, n'est pas inférieure à celle qu'ont les concepts théoriques et opératoires, comme le principe des espaces servants et servis. L'épreuve de l'échelle du territoire, l'usage de la pente et la notion de la façade sont ainsi les uns de plusieurs principes sur lesquels Louis Kahn fonde sa méthode, et ils évoluent au fil de l'expérience professionnelle de l'architecte.

\section{DISCUSSION}

Étant une réponse à la crise de l'architecture civique dans l>après-guerre, le placemaking de Louis Kahn est promoteur de ces tendances postmodernes qui s'opposent à la culture de masse. Tout d'abord, la volonté de restituer la signification sociale de l'espace public pousse certains architectes à la recherche d'une nouvelle monumentalité. L'importance qu'un groupe d'architectes de l'après-guerre attribue au monument d'architecture et à sa capacité de représenter l'identité sociale fait référence à l'application du modèle linguistique dans le champ des sciences humaines. Se développant sur cette base, la théorie de la sémantique de l'espace urbain s'enrichit du concept de repère (ang. landmark). Souvent attribué à une institution, le rôle de repère urbain inspire la quête de la nouvelle monumentalité. Conjointement, naît la notion de lieu avec lequel les hommes peuvent s'identifier. Déjà dans les années cinquante, les architectes britanniques Alison et Peter Smithson prennent comme objectif la restitution du lieu, ce qu'ils pensent nécessaire pour «relier de nouveau l'homme à sa maison, à sa communauté, à sa ville». [Ksiazek, 1993, p.419]

Cet intérêt porté au monument, compris comme un repère urbain et social, s'accompagne de la naissance de deux modèles esthétiques: le nouvel humanisme et le régionalisme. [Ksiazek, 1993] Tandis que le premier recourt au langage de la Renaissance italienne dans lequel il reconnait le potentiel de restituer à l'architecture sa valeur institutionnelle, le second cherche la source d'inspiration dans la spécificité de la région. Fréquemment cité pour l'intérêt qu'il porte au langage classique hérité de sa formation Beaux-Arts, Louis Kahn s'inspire également du caractère particulier de la région. Tout en fondant son vocabulaire sur les principes classiques de composition, l'architecte s'ouvre aux influences locales pour enrichir sa méthode et pour relier ses projets au contexte. Et, à travers une longue et fine étude, il sait intégrer les grands types architecturaux dans l'environnement contemporain. Enraciné dans l'époque marquée par la recherche d'une nouvelle monumentalité, le placemaking de Louis Kahn est ainsi imprégné tant du nouvel humanisme que du régionalisme.

Issu de cette dernière tendance et proche de la démarche de Louis I. Kahn est le régionalisme critique. Prôné par Frampton, Tzonis et Lefaivre, ce courant cherche à unir l'apport du Mouvement moderne avec l'héritage local. Tout en s'opposant à l'imitation exacte des formes de l'architecture vernaculaire, il s'inspire des conditions caractéristiques du lieu: de son climat et de la lumière, mais aussi de son héritage culturel et bâti, y compris le choix des matériaux. Le régionalisme critique se met pour objectif d'élaborer «une synthèse consciente de la civilisation universelle et de l'héritage culturel du monde». [Frampton, 1983] Dans cet équilibre réside la conception d'un lieu identifiable, défini par Frampton comme place-form. S'opposant 
au développement infini d'une structure urbaine indifférente, associée au concept de mégalopole, le lieu constitue un repère non seulement urbain, mais aussi en termes de conscience sociale. Le rôle que joue la mémoire collective dans le concept de lieu est souligné par Aldo Rossi. D'après sa vision, le lieu se définit non seulement à travers son espace-temps, mais aussi par son histoire humaine: «Et donc ce qui fait du locus même un fait singulier déterminé par l'espace et le temps, par sa dimension topographique et par sa forme, par le fait d'être le lieu d'une histoire ancienne et récente, par sa mémoire». [Rossi, 2001, p.145]

Nourri de l'expérience de Louis I. Kahn, le régionalisme critique développe la notion de lieu. Supposant l'intégration de l'architecture à son environnement, le concept s'enrichit d'un élément humain important, ce qui est la mémoire collective. Étant des espaces inscrits dans la conscience de ses habitants, les lieux constituent une réponse que certains architectes de l'après-guerre adressent à la crise de l'identité sociale.

\section{BIBLIOGRAPHIE}

Brownlee D., De Long D., 1991. Louis I. Kahn. In the Realm Of Architecture, Rizzoli, New York.

Frampton K., 1983. Towards a Critical Regionalism: Six Points for an Architecture of Resistance, [dans:] The Anti-Aesthetic, Essays on Postmodern Culture, réd. Foster H., The New Press, New York.

Goldhagen S. Williams, 2001. Louis Kahn's Situated Modernism, Yale University Press, New Haven, CT.

Komendant A.E., 1975. 18 years with architect Louis I. Kahn, Aloray Publisher, Englewood, NJ.

Ksiazek S., 1993. Architectural Culture in the Fifties: Louis Kahn and the National Assembly Complex in Dhaka, [dans:] Journal of the Society of Architectural Historians, Vol. 52, No. 4.

Latour A., 1991. Louis I. Kahn: Writings, lectures, interviews, Rizzoli, New York.

Lyndon D., Moore Ch.W., 1994. Chambers for A Memory Place, with illustrations by the authors, The MIT Press, Cambridge, MA.

Ronner H., Jhaveri Sh., 1987. Louis I. Kahn. Complete Work 1935-74, Second Revised and Enlarged Edition, Birkhäuser, Basel.

Rossi A., 1966. L'architettura della città, (trad. fr. L'Architecture de la ville, Infolio éditions, Gollion 2001).

Venturi R., 1996. Iconography and Electronics. Upon a Generic Architecture. A View from the Drafting Room, The MIT Press, Cambridge MA. 


\section{ILLUSTRATIONS}
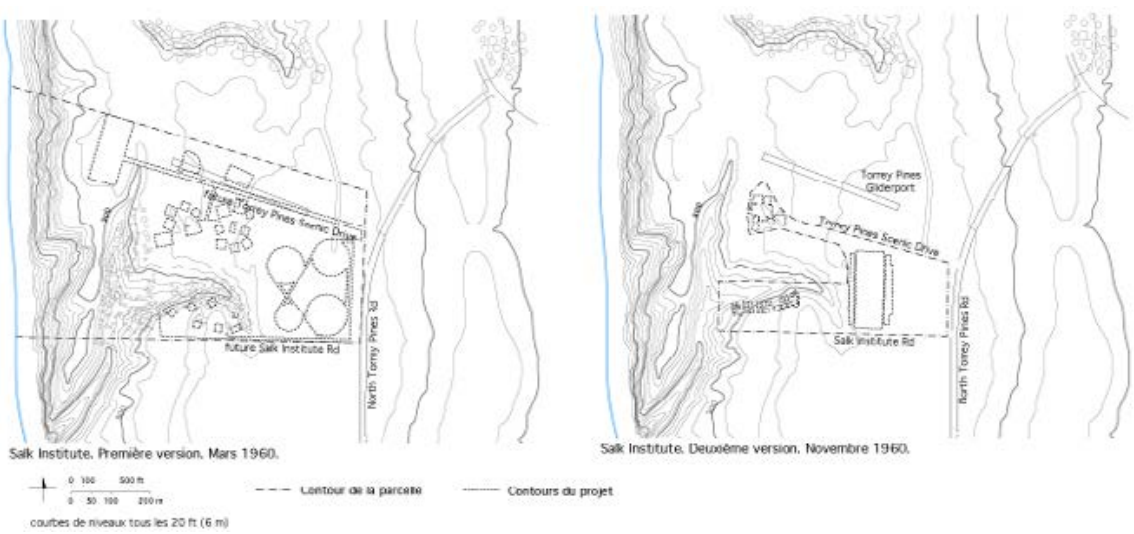

Ill. 1. Salk Institute, La Jolla CA. L'appropriation de l'échelle du site au travers de phases du projet. Ryc. 1. Instytut Salka, La Jolla CA. Przyswojenie skali miejsca poprzez kolejne fazy projektu.
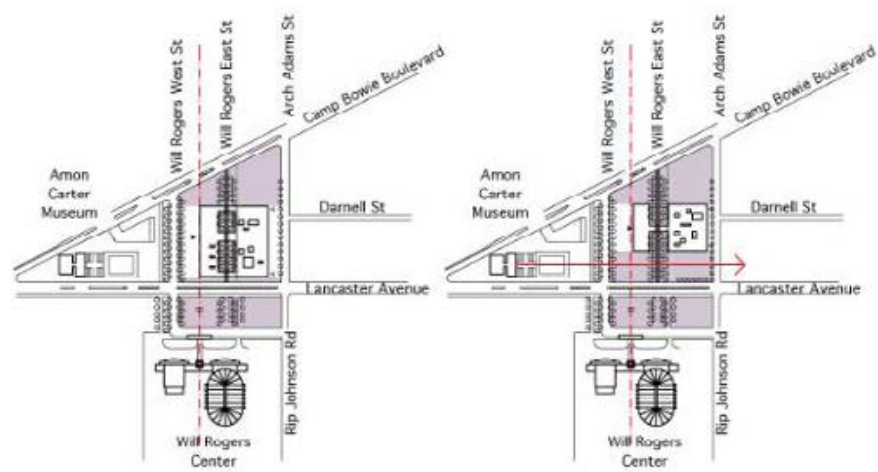

1. Première version, Square-Plan 1966 - 1967

2. Deuxième version,

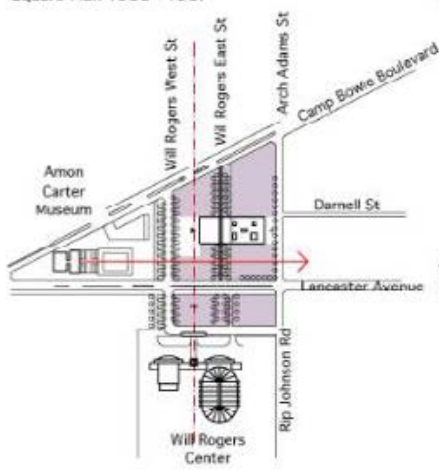

H.Plan 1967 - aoút 1968

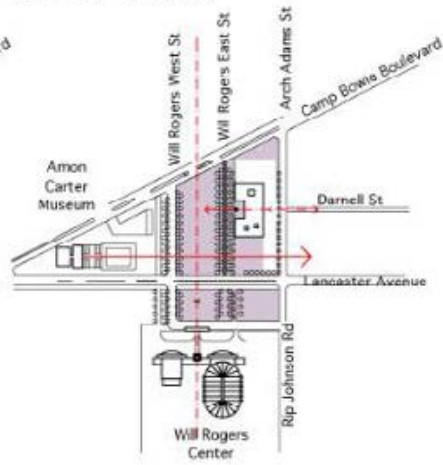

3. Version de transition,

C-Plan aoút $1968-1969$

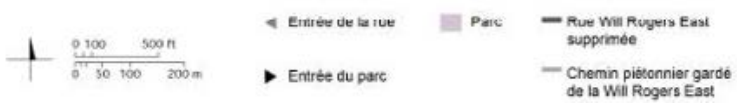

Ill. 2. Musée Kimbell, Fort Worth TX. L'appropriation de l'échelle du site au travers de phases du projet. Ryc. 2. Muzeum Kimbell, Fort Worth TX. Przyswojenie skali miejsca poprzez kolejne fazy projektu. 


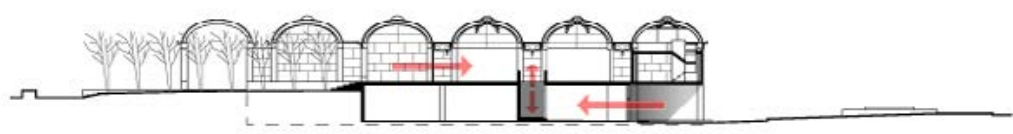

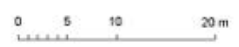

Ill. 3. Musée Kimbell, Fort Worth TX. Version finale, CPlan. Coupe transversale montrant les deux entrées et la distribution principale.

Ryc. 3. Muzeum Kimbell, Fort Worth TX. Wersja ostateczna projektu, tzw. CPlan. Przekrój poprzeczny ukazujący dwa wejścia oraz główną oś dystrybucji.

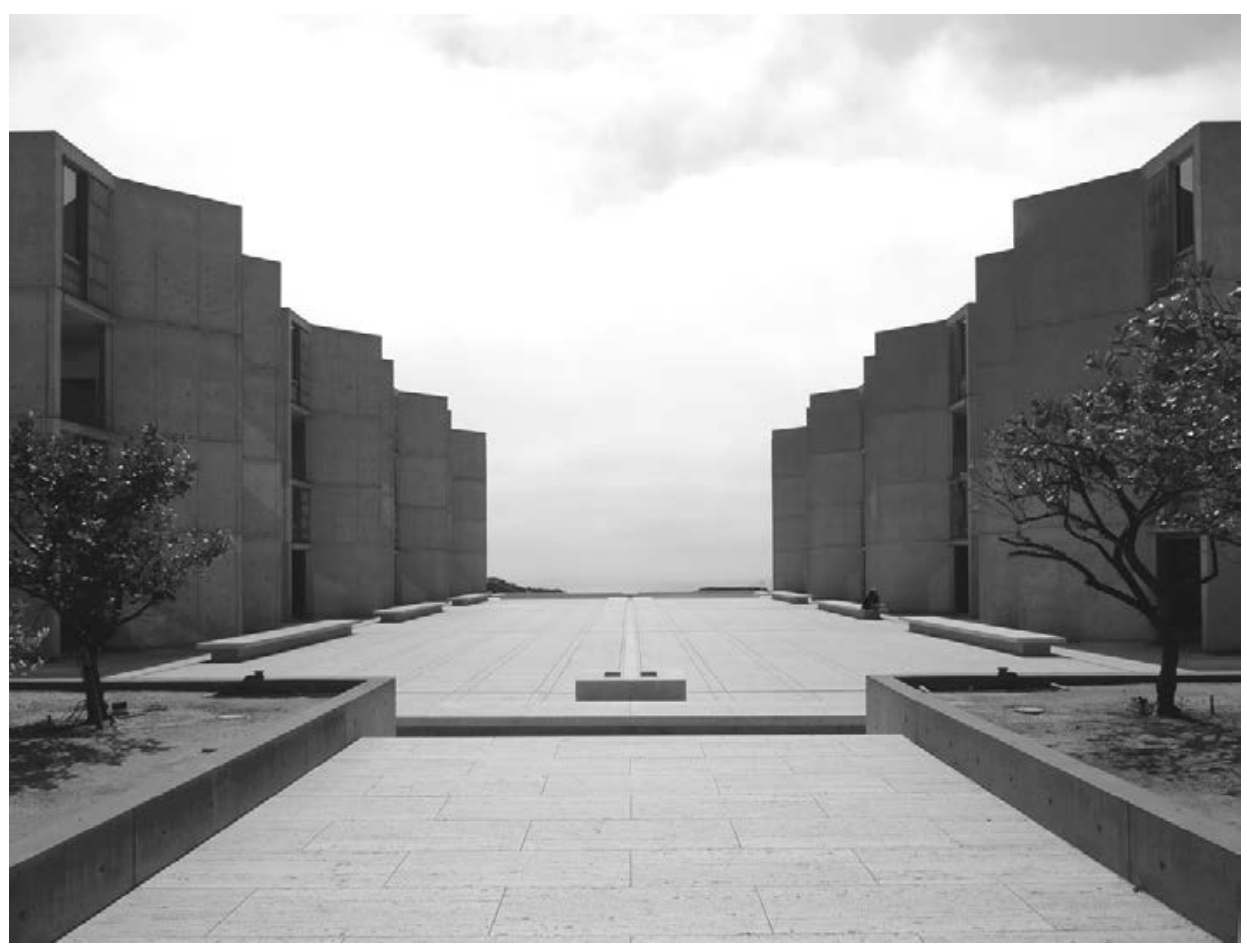

Ill. 4. Salk Institute, La Jolla CA. Plaza centrale. Théâtralisation du paysage. Ryc. 4. Instytut Salka, La Jolla CA. Plac centralny. Teatralizacja krajobrazu.

\section{PLACEMAKING WEDŁUG LOUISA I. KAHNA}

Streszczenie. Definiowany jako „tworzenie miejsc”, placemaking opiera się nie tylko na stosowaniu wyszukanego języka architektonicznego, ale również na harmonijnym połączeniu architektury z jej miejscem położenia. W twórczości Louisa I. Kahna, rola jaką w jego projektach odgrywa teren i otoczenie nie ogranicza się jedynie do wpływu na formę pojedynczych budowli, ale przyczynia się jednocześnie do rozwoju jego indywidualnej metody pracy. Rożne miejsca, lokalizacje, w których Kahn osadza swoje kolejne budowle stanowią źródło uniwersalnych refleksji związanych z wykonywaniem zawodu architekta. W ich efekcie Louis I. Kahn wypracował na przestrzeni swojej kariery szereg zasad projektowych, które nazwać możemy jego „narzędziami placemaking'u” i które nierozerwalnie składają się na jego metodę projektową.

Slowa kluczowe: placemaking („tworzenie miejsc”), miejsce, teren, kontekst urbanistyczny, metoda pracy, realizacja projektu. 